\title{
BOUNDARY LAYER CONTROL OF A RADIATIVE CASSON FLUID FLOW PAST A PERMEABLE RIGA-PLATE WITH UNIMOLECULAR CHEMICAL REACTION
}

\author{
P. LOGANATHAN ${ }^{\dagger}$ and K. DEEPA ${ }^{\ddagger}$ \\ Department of Mathematics, Anna University, Chennai, Tamilnadu, 600025, India. \\ $\dagger$ †ogu@annauniv.edu, $¥$ deepakkrish1@gmail.com
}

\begin{abstract}
The buoyancy driven, chemically reacting and radiative Casson fluid flow past an impulsively started permeable Riga-plate is investigated through the numerical solution obtained by CrankNicholson implicit scheme of finite difference method. The main aim of this study is to control the boundary layer separation. Escalating modified Hartmann number and the distance from leading edge of the plate reduces the viscous drag so that the separation can be controlled. Effects of permeability on the flow configuration are also elucidated. The results are validated by comparing the solutions of literature which already exists.
\end{abstract}

Keywords - Riga-plate; Electromagnetism; Casson fluid; EMHD Lorentz force; permeability.

\section{INTRODUCTION}

The applied external magnetic field has significant impact on highly conducting fluids; however the weakly conducting fluids induce only less amount of current. As a result, an external magnetic field should be applied to achieve a powerful control in boundary layer separation (Magyari and Pantokratoras, 2011). The classical methods suction, blowing and motion of the wall hinder the separation of the boundary layer from the surface. This irregular flow behavior can be controlled by electromagnetism, which provides convincing flow control on account of high electrical conductivity of the material (Weier et al., 2001).

Gailitis and Lielausis from physics institute at Riga designed an electromagnetic control system which is a span wise alternate arrangement of electrodes and permanent magnets. This actuator is termed as Riga-plate. The influence of EMHD Lorentz force on free convection over Riga-plate is studied by Pantokratoras and Magyari (2009). Magyari and Pantokratoras (2011) analyzed doubly driven aiding and opposing flow characteristics using finite difference scheme numerically. It was observed that reverting flow displays reduction in the velocity. Nanofluid flow on a Riga-plate is also examined by many researchers in recent years. Various numerical solutions for nanofluid flow past a Riga-plate has been obtained by Abbas et al. (2017), Ayub et al. (2016), Rasool and Zhang (2019). Hayat et al. (2018) found the homotopic solution for a mixed convective fluid subject to radiation and double stratification on a Riga-plate. Pantokratoras (2008) estimated the exact solution of Blasius-Sakiadis flow with MHD Lorentz force or EMHD Lorentz force.
Non-Newtonian fluid characteristic is observed in many chemical and allied processing industries. Determining the rheological behavior of such material is highly complex. These fluids are highly consistent which can be processed under laminar conditions. A constitutive relation which was found by Casson delineates the relationship between shear stress and rate of deformation. Cone-plate viscometer was used to exemplify this empirical equation, when the pigments suspended in varnishes. This fluid is termed as Casson fluid (Oka, 1981).

Owing to the broad range of applications, much of literature and studies are directed towards the Casson fluid flow model. Rao (2018) obtained the numerical solution using shooting technique for Casson fluid flow past a permeable stretching surface with thermal and mass diffusion. A mathematical model for the Casson fluid flow with periodic variations in a porous channel was analyzed by Srinivas et al. (2018). Perturbation technique is used to obtain the analytical solution in this investigation.

Reza et al. (2016) examined the influence of suction/blowing on Casson fluid flow past a stretching surface. A remarkable conclusion in this study is that the speed and heat transfer of the flow regulated substantially because of suction and injection on the wall. Iqbal et al. (2017) centralized their priority to viscous dissipative and thermally radiative Casson fluid flow past a Rigaplate subject to internal heat generation. The flow control attributes of Reiner-Phillipoff model and Powel Erying model over Riga-plate are scrutinized by Ahmad (2019).

In real time operations, electro-magnetic propulsion systems are distinct mechanisms from typical drive components that are used in ship propulsion. The current which is passing through the fluid and involved in an externally applied magnetic force produces Lorentz force; this phenomenon results in the propulsion of vessel to move forward. EMHD is also used as a recovery tool for oil spill on sea water by the action of pumping (AlHabahbeh et al., 2016). In some scenario, EMHD is a therapeutic remedy to regulate the poor blood flow. Casson fluid flow model is the most suitable non Newtonian model for describing the characteristics of human and cattle blood. The theoretical investigation associated with EMHD Casson fluid with moving boundary may also be considerate in the pumping of blood flow to modulate its circulation.

This numerical investigation intended towards the mechanical properties of Casson fluid past a vertically moving permeable Riga-plate. The transient flow configuration is constructed for a radiative Casson fluid subject 
to both MHD and EMHD Lorentz force with unimolecular chemical reaction. A comparison study has been performed to ensure the accuracy of the present results.

\section{MATHEMATICAL ANALYSIS}

Consider a gravitationally driven chemically reacting and radiative Casson fluid flow over a permeable impulsively started vertical Riga- plate. The semi-infinite vertical plate is setting idle for an initial stage and the ambient temperature $T_{\infty}^{*}$, concentration $C_{\infty}^{*}$ are persisted by the fluid. The plate is actuated vertically upwards with a constant speed $u_{0}$ at $t^{*}>0$. Temperature of the plate is increased to $T_{w}^{*}\left(>T_{\infty}^{*}\right)$, and concentration near the plate surface is boosted up to $C_{w}^{*}\left(>C_{\infty}^{*}\right)$. The temperature and concentration reaches the asymptotic state and the velocity diminishes to zero while the fluid flow away from the surface. The coordinate system is constructed on the basis of fluid motion. The vertical fluid motion is entitled as $x$ direction and the $y$ direction is normal to the plate. $u$ and $v$ are the velocities along vertical and transverse directions respectively. Optically thick and non scattering conditions are considered. The homogeneous first order chemical reaction is under consideration. The Riga-plate setup has been illustrated in Fig 1.

The flow configuration of Casson fluid can be expressed by the following constitutive relation (Mustafa et al., 2014; Shateyi et al., 2017)

$$
\tau_{i j}=\left\{\begin{array}{l}
2\left(\mu_{b}+P_{y} / \sqrt{2 \pi^{*}}\right) e_{i j}, \pi^{*}>\pi_{c}^{*} \\
2\left(\mu_{b}+P_{y} / \sqrt{2 \pi_{c}^{*}}\right) e_{i j}, \pi^{*} \leq \pi_{c}^{*}
\end{array} .\right.
$$

Here, $\pi^{*}=e_{i j} e_{i j}$ and $e_{i j}$ is the $(i, j)^{\text {th }}$ component of the shear rate. The physical problem governed by the Prandtl boundary layer equations that evince the above conditions, constitutive equation and Boussinesq approximation can be described as (Schlichting, 1979)

$$
\begin{gathered}
\frac{\partial u}{\partial x}+\frac{\partial v}{\partial y}=0 \\
\frac{\partial u}{\partial t^{*}}+u \frac{\partial u}{\partial x}+v \frac{\partial u}{\partial y}=v\left(1+\frac{1}{\beta}\right) \frac{\partial^{2} u}{\partial y^{2}}+g \beta^{\prime}\left(T^{*}-T_{\infty}^{*}\right)+ \\
g \beta^{*}\left(C^{*}-C_{\infty}^{*}\right)+\frac{\pi J_{0} M_{0} e^{-\pi y / l}}{8 \rho}-\frac{\sigma B_{0}^{2} u}{\rho}-\frac{u v}{\lambda^{*}} \\
\frac{\partial T^{*}}{\partial t^{*}}+u \frac{\partial T^{*}}{\partial x}+v \frac{\partial T^{*}}{\partial y}=\alpha \frac{\partial^{2} T^{*}}{\partial y^{2}}-\frac{1}{\rho C_{p}} \frac{\partial q_{r}}{\partial y} \\
\frac{\partial C^{*}}{\partial t^{*}}+u \frac{\partial C^{*}}{\partial x}+v \frac{\partial C^{*}}{\partial y}=D \frac{\partial^{2} C^{*}}{\partial y^{2}}-k^{\prime}\left(C^{*}-C_{\infty}^{*}\right)
\end{gathered}
$$

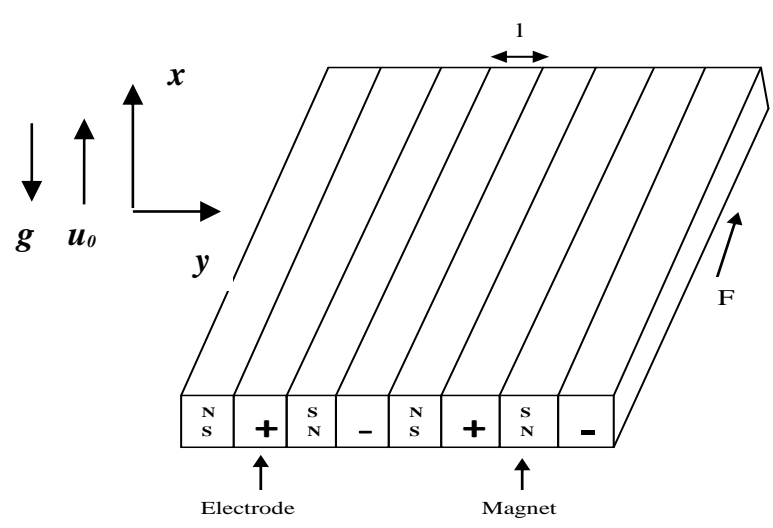

Fig. 1. Arrangement of electrodes and magnets in Riga-plate.
Preferable initial and boundary conditions of the physical problem are

$t^{*} \leq 0, u=0, v=0, T^{*}=T_{\infty}^{*}, C^{*}=C_{\infty}^{*}$ for all $x$ and $y$,

$$
\begin{gathered}
t^{*}>0, u=u_{0}, v=0, T^{*}=T_{w}^{*}, C^{*}=C_{w}^{*} \text { for } y=0, \\
u=0, v=0, T^{*}=T_{\infty}^{*}, C^{*}=C_{\infty}^{*} \text { for } x=0, \\
u \rightarrow 0, T^{*} \rightarrow T_{\infty}^{*}, C^{*} \rightarrow C_{\infty}^{*} \text { as } y \rightarrow \infty .
\end{gathered}
$$

Here $\beta=\mu_{b} \sqrt{2 \pi_{c}^{*}} / P_{y}$ is the Casson parameter. In the energy balance equation, $q_{r}=-4 \sigma^{*} / 3 a^{\prime} \partial T^{* 4} / \partial y$ denotes the Rosseland approximation (Brewster 1992).

Non dimensional quantities employed on the coupled highly non-linear partial differential Eqs. (1-4) are taken as

$$
\begin{gathered}
X=\frac{x u_{0}}{v}, Y=\frac{y u_{0}}{v}, U=\frac{u}{u_{0}}, V=\frac{v}{u_{0}}, t=\frac{t^{*} u_{0}^{2}}{v}, \\
T=\frac{T^{*}-T_{\infty}^{*}}{T_{W}^{*}-T_{\infty}^{*}}, C=\frac{C^{*}-C_{\infty}^{*}}{C_{W}^{*}-C_{\infty}^{*}}, G r=\frac{v g \beta^{\prime}\left(T_{W}^{*}-T_{\infty}^{*}\right)}{u_{0}^{3}}, \\
G C=\frac{v g \beta^{*}\left(C_{W}^{*}-C_{\infty}^{*}\right)}{u_{0}^{3}}, \operatorname{Pr}=\frac{v}{\alpha}, N=\frac{\kappa a^{\prime}}{4 \sigma^{*} T_{\infty}^{* *}}, \lambda=\frac{\lambda^{*} u_{0}^{2}}{v^{2}}, \\
M=\frac{\sigma B_{0}^{2} v}{\rho u_{0}^{2}}, K^{*}=\frac{k^{\prime} v}{u_{0}^{2}}, S c=\frac{v}{D}, P=\frac{\pi J_{0} M_{0} v}{8 \rho u_{0}^{3}}, S=\frac{\pi v}{l u_{0}} . \\
\text { Dimensionless form of }(1-5) \text { are given by } \\
\frac{\partial U}{\partial t}+U \frac{\partial U}{\partial X}+V \frac{\partial U}{\partial Y}=\left(1+\frac{\partial V}{\beta}\right) \frac{\partial^{2} U}{\partial Y^{2}}+G r T+G c C+ \\
P e^{-S Y}-M U-\frac{U}{\lambda}, \\
\frac{\partial T}{\partial t}+U \frac{\partial T}{\partial X}+V \frac{\partial T}{\partial Y}=\frac{1}{\operatorname{Pr}}\left(1+\frac{4}{3 N}\right) \frac{\partial^{2} T}{\partial y^{2}}, \\
\frac{\partial C}{\partial t}+U \frac{\partial C}{\partial X}+V \frac{\partial C}{\partial Y}=\frac{1}{S c} \frac{\partial^{2} C}{\partial Y^{2}}-K^{*} C .
\end{gathered}
$$

Initial and boundary conditions in terms of non-dimensional quantities are

$$
\begin{gathered}
t \leq 0, U=0, V=0, T=0, C=0 \text { for all } X \text { and } Y, \\
t>0, U=1, V=0, T=1, C=1 \text { for } Y=0, \\
U=0, V=0, T=0, C=0 \text { for } X=0, \\
U \rightarrow 0, T \rightarrow 0, C \rightarrow 0 \text { as } Y \rightarrow \infty .
\end{gathered}
$$

\section{NUMERICAL SOLUTION}

Solutions of two dimensional non-linear partial differential Eqs. (6-9) together with the initial and boundary conditions (10) are acquired by finite difference scheme, namely Crank-Nicholson implicit method. The solution procedure starts with the discretization of the above system of partial differential equations. Each term transformed to a finite difference approximation of corresponding order. The approximation converts the system to a set of linear algebraic difference equations. The coefficient matrix of this system is a tri-diagonal matrix.

Using tri-diagonal matrix algorithm, velocity $U$, temperature $T$ and concentration $C$ were obtained (Carnahan et al., 1969). The uniform grid spacing $\Delta X$ and $\Delta Y$ with $\Delta t$ time steps are considered. The values of $X$ and $Y$ assigned to $i \Delta X$ and $j \Delta Y$, respectively. Domain of integration is a rectangle with $X_{\max }=1.0$ and $Y_{\max }=14.0$. Here $Y_{\max }$ describes the value of $Y$ at $\infty$ which is far away from the momentum, thermal and concentration boundary layer. The truncation error approaches zero as $\Delta X, \Delta Y$ and $\Delta t \rightarrow 0$. The scheme is unconditionally stable and convergent. After obtaining velocity, temperature and 
concentration the other flow characteristics skin friction, Nusselt number and Sherwood number can be evaluated by the following expressions

$$
\begin{gathered}
\tau_{X}=-\left(1+\frac{1}{\beta}\right)\left(\frac{\partial U}{\partial Y}\right)_{Y=0}, \bar{\tau}=-\int_{0}^{1}\left(1+\frac{1}{\beta}\right)\left(\frac{\partial U}{\partial Y}\right)_{Y=0} d X, \\
N u_{X}=-X\left(\frac{\partial T}{\partial Y}\right)_{Y=0}, \overline{N u}=-\int_{0}^{1}\left(\frac{\partial T}{\partial Y}\right)_{Y=0} d X, \\
S h_{X}=-X\left(\frac{\partial C}{\partial Y}\right)_{Y=0}, \overline{S h}=-\int_{0}^{1}\left(\frac{\partial C}{\partial Y}\right)_{Y=0} d X .
\end{gathered}
$$

\section{RESULTS AND DISCUSSION}

To estimate the reliability of the current results, comparison of velocity has been done with the already available study of Pantokratoras and Magyari (2009). This is made under the conditions that the plate is stationary and $\lambda, \beta \rightarrow$ $\infty, G r=G c=M=0, P=S=1$. Pantokratoras and Magyari have given an extensive steady state analysis of EMHD effects on a horizontal Riga-plate with the only driving force as electromagnetic body force. Precisely, the work is focused on the study of the skin friction and the velocity along the different distances from the leading edge. The non uniform grid spacing is taken into consideration while finding solutions by finite difference method. The present investigation is imperatively driven by the buoyancy force over a vertically oriented Riga-plate and the thermal, mass diffusion behaviour has also been discerned under unsteady circumstances. However, the discussions are carried out for the achieved steady state solutions. Besides, the boundary layer equations are solved with uniform step sizes.

The values of temperature and concentration are validated with the solutions of Gebhart and Pera (1971) for $\operatorname{Pr}=S c=0.7, G r=G c=2, K^{*}=0, P=0, M=0, N, \lambda, \beta \rightarrow \infty$. The investigation which was done by Gebhart and Pera focused on the influence of natural convection on vertical flow. The exactness and excellent correlation of the analysis is ensured in Fig. 2.

Figure 3 depicts the influence of pertinent parameters on velocity field. For $P>0$, speed of the flow increases due to the fluid flow along the main stream velocity. This refers to the aiding flow characteristics of the fluid. How-

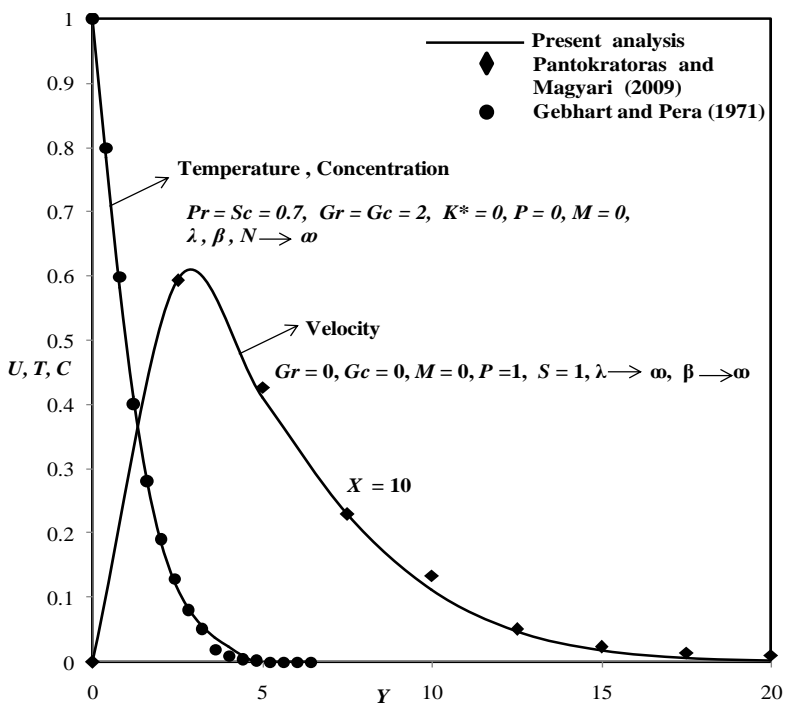

Fig. 2. Correlation of flow properties.

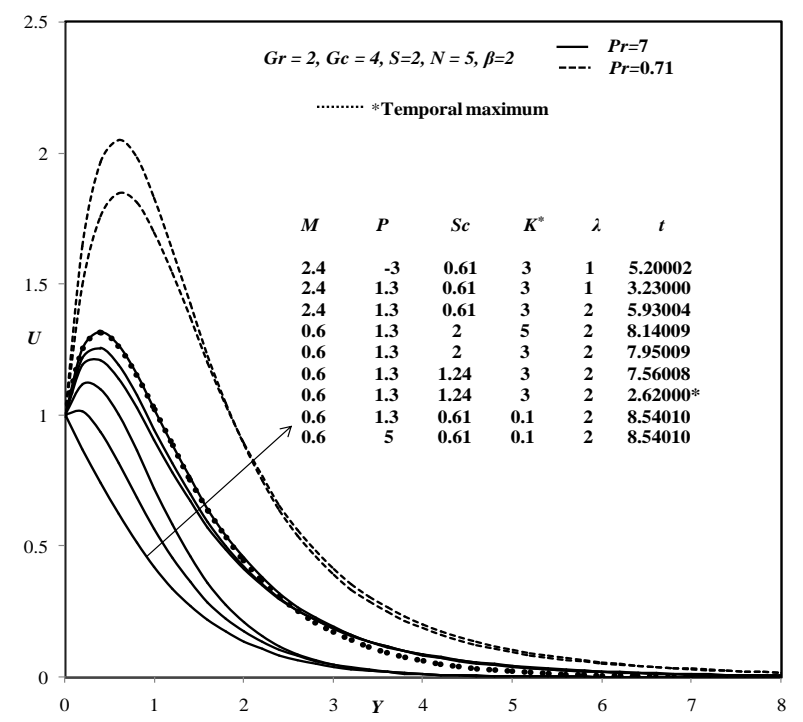

Fig. 3. Steady state velocity profiles at $X=1.0$.

ever, the fluid encounters the additional viscosity in the case of opposing flow $(P<0)$ which results in the velocity drop and the steady state reaches at the elongated time level. Uplifting the permeability parameter $\lambda$ leads to the growth in the velocity boundary layer. Owing to higher viscosity, the fluid flow experiences the resistance for increased values of $S c$. Velocity of the fluid decays in respect of the reactant, i.e. rise in the values of chemical reaction parameter $K^{*}$ slow down the speed. The velocity reaches the steady state at the larger time level with the increased values of $\lambda, S c$ and $K^{*}$. The time at which the velocity attains its steady state temporarily (temporal maximum) is also displayed.

Figure 4 displays the aiding and opposing flow properties of Casson fluid at different distances from the leading edge of the plate. Aiding flow which is along the direction of main stream velocity encounters the wall parallel Lorentz force that stimulates the flow velocity. A reasonable drop in the velocity is noticed (comparing to the aiding flow) when the Lorentz force acting opposite

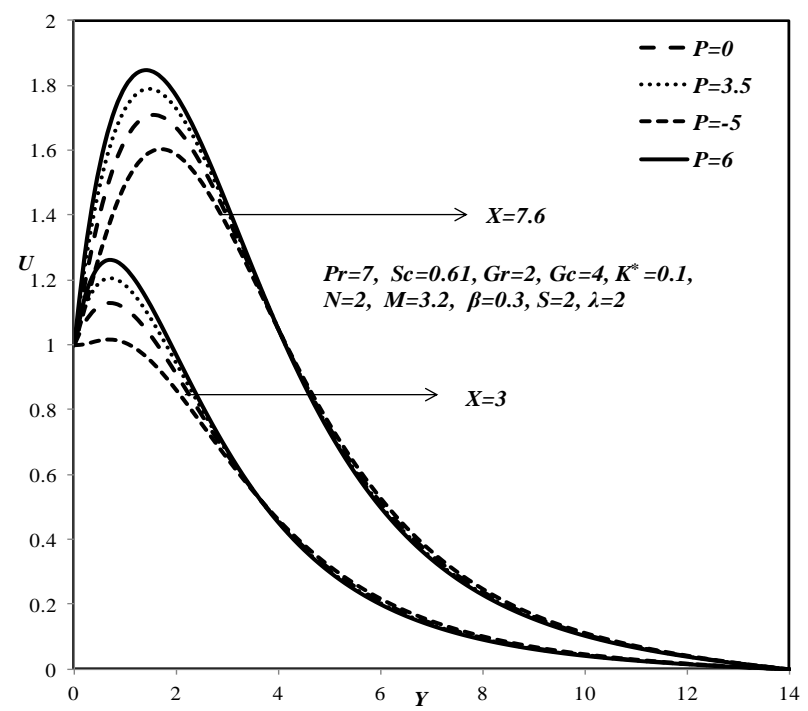

Fig. 4. Aiding and opposing flow characteristics. 


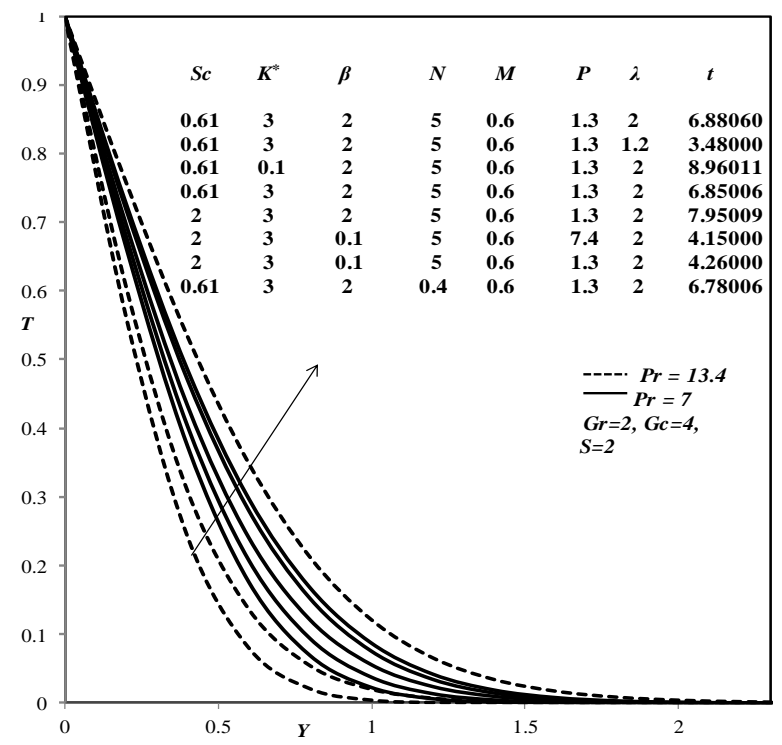

Fig. 5. Temperature field for various parameters.

to the flow direction. This is achieved by upturned the electrode's polarity. However, the viscous drag is reduced in both the cases. When the distance from the plate and the values of $P$ are increased, the flow is not more prone to boundary layer separation. This implies that the separation can be controlled by the elevation of modified Hartmann number and the distances from the edge.

The temperature dependent physical properties of Casson fluid are illustrated in Fig. 5. Temperature drop is observed for the improved values of Prandtl number owing to less physical strength of the thermal diffusivity. The lower molecular diffusion rate of heightened $S c$ deteriorates temperature distribution. Elevation in the chemical reaction rate $\left(K^{*}\right)$ between the fluid molecules enhances the temperature. Radiation controls the electron transport in the magnets, electrodes. This implies that the thermal energy declines for the raise in radiation parameter $N$. Temperature shoots up for the increased permeability. On the other hand, elevated Casson parameter reduces the thermal diffusion. The modified Hartmann number $P$ depends on the current density and magnetization of the magnets. Raising the values of $P$ results in the escalation of magnetization. Thus, the magnetic field strength reduces the temperature. In respect of heightened radiation parameter speeds up time to attain steady state.

In Fig. 6, the mass diffusion is displayed as a function of $Y$ for the fixed values of some chosen physical parameters. Elevated chemical reaction parameter increases the reaction rate which in turn worsens the growth of the concentration boundary layer. While escalating the values of $S c$, it causes the lower mass diffusivity that tends to reduce the mass diffusion in the species. The heightened values of $\operatorname{Pr}, N, M$ and $\beta$ enhances the mass diffusion. Exalting modified Hartmann number $P$, the deterioration is noticed in concentration subject to stronger Lorentz force. Time taken to reach the steady state increases for increased chemical reaction parameter, whereas it decays for Schmidt number.

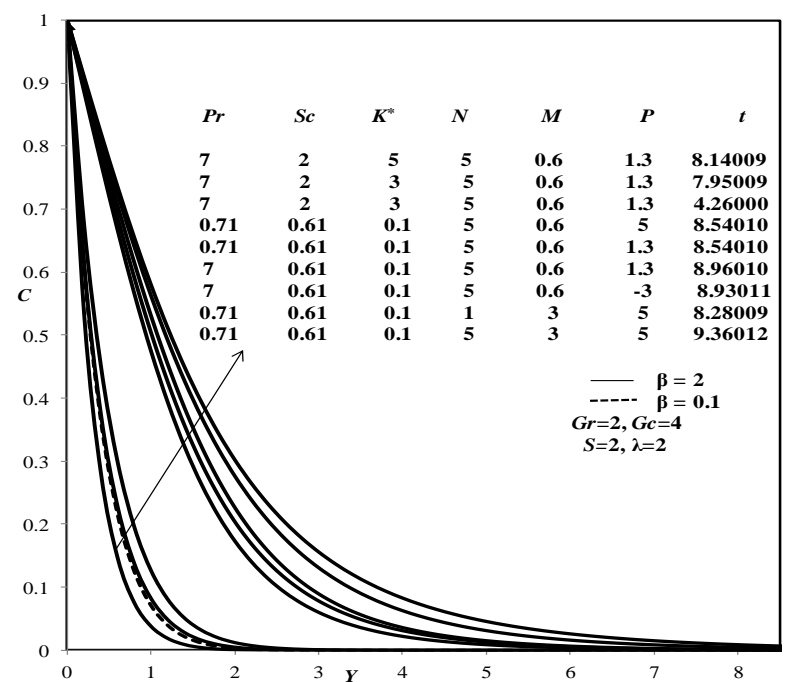

Fig. 6. Concentration distribution.

The local and mean skin friction coefficient has been analyzed in Figs. 7 and 8 respectively. Changes in the velocity gradient with respect to viscosity and yield stress reports the skin friction on the surface. Raise in the values of Prandtl number affects the fluid motion with the high viscosity that creates the retardation in the flow i.e. the higher skin friction is observed. When $K^{*}$ is increased, concentration of the reactant is enhanced. This phenomenon boost up the friction between the fluid particles. Due to the bulk behavior of the fluid molecules, the fluid flow can easily approach the plate for bigger pores or large number of pores. This implies that the skin friction is higher for exalted $\lambda$. Uplifting the values of $P$, the EMHD Lorentz force displays the lowest skin friction. The magnetic parameter and Schmidt number enhances the viscous drag locally on account of MHD Lorentz force and heightened viscosity respectively.

Variations in the local and average rate of heat transfer explained in Figs. 9 and 10. Enhanced values of $K^{*}$, slow down the heat transfer by virtue of high concentration of the reactant. Improved radiation parameter escalates the heat transfer from high energy region to low en-

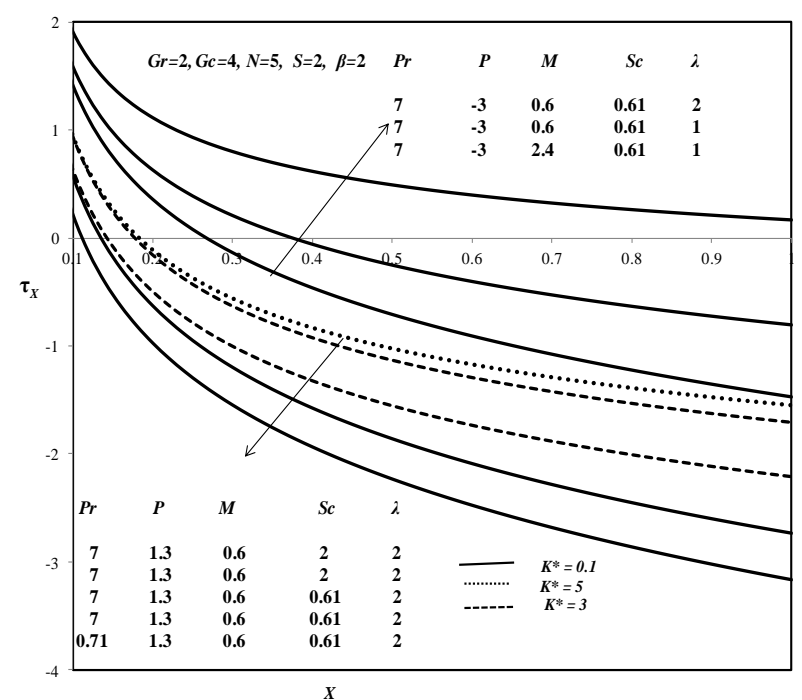

Fig. 7. Local skin friction coefficient. 
ergy region. As a consequence of EMHD Lorentz force, the heat transfer boost up proportionately to the values of

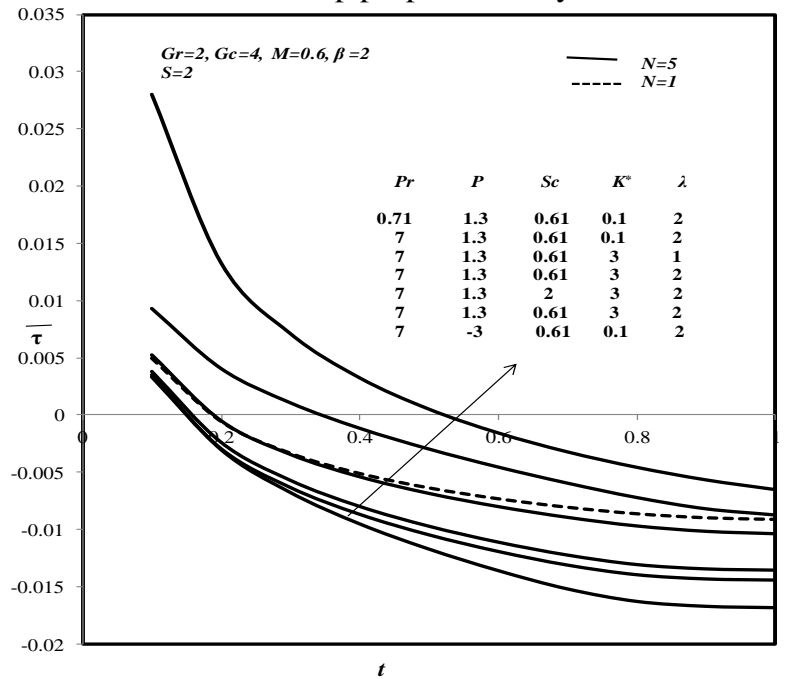

Fig. 8. Mean skin friction coefficient.

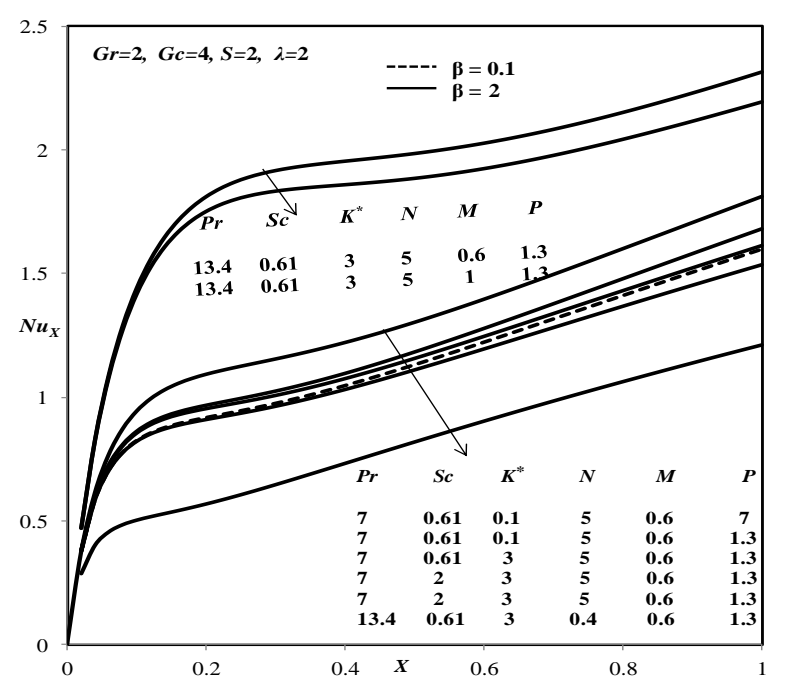

Fig. 9. Local Nusselt number.

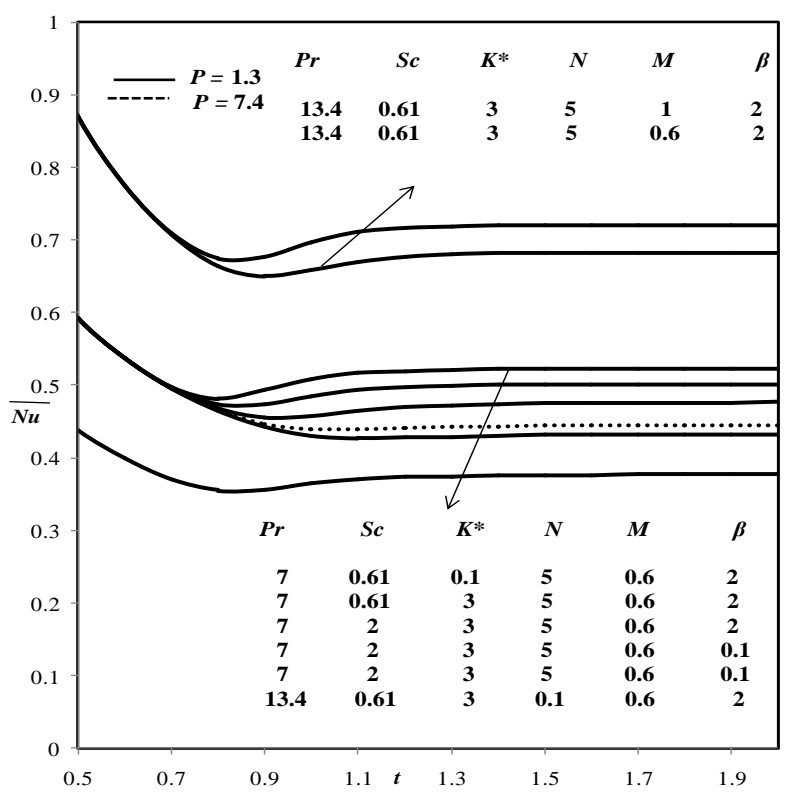

Fig. 10. Mean Nusselt number. modified Hartmann number. The plastic dynamic viscosity of increased Casson parameter weakens the heat transfer. As the magnetic field has a control on the heat transfer between fluid particles, higher values of $M$ declines the heat transfer rate. Larger Prandtl number values enhance the temperature gradient which results in the uplifted heat transfer rate.

The rate of heat and mass transfer inside the boundary layer is explored through the variations of temperature and concentration gradients encountered in the fluid flow. The Nusselt number and Sherwood number which explains theses variations has been discussed along the plate surface. The average of gradients at each time level induces the case of thermal and mass transport in an average sense.

Figures 11 and 12 presents the local and average Sherwood number. As a result of reduction in mass diffusivity while increasing $S c$, the improved concentration gradient is observed which boost up the values of Sherwood number. The high concentration of the reactant due to heightened $K^{*}$ strengthens the rate of mass transfer. The improved modified Hartmann number shoots up the mass transfer rate locally.

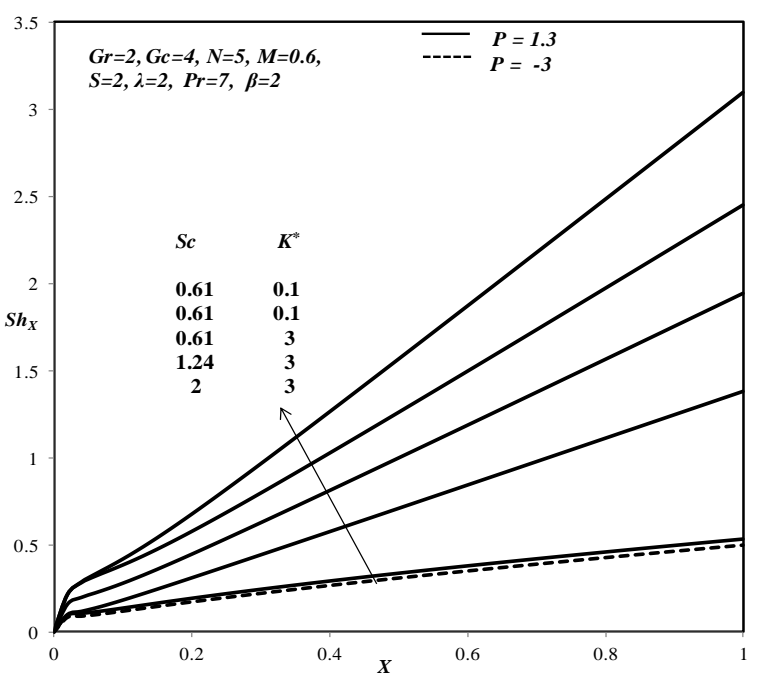

Fig.11. Local Sherwood number.

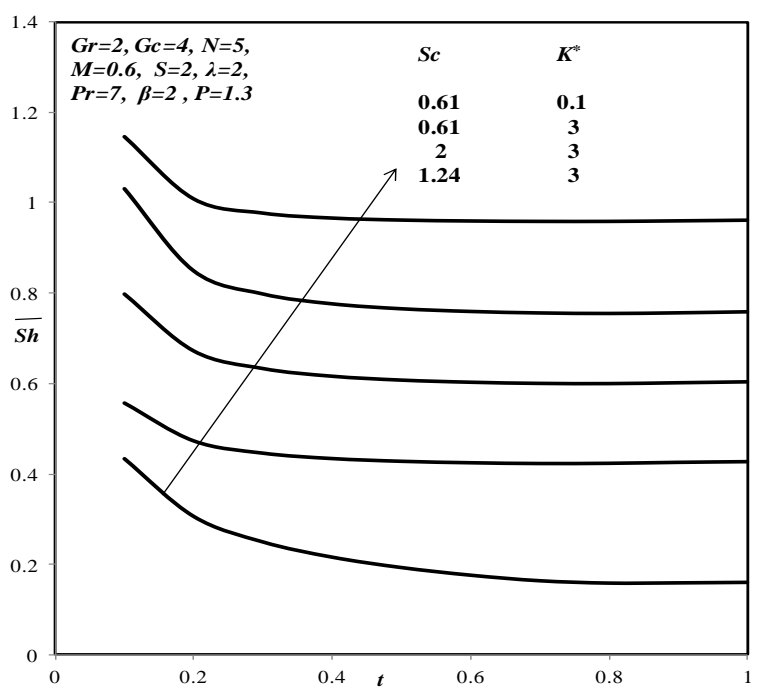

Fig. 12. Mean Sherwood number. 


\section{CONCLUSIONS}

Many investigations centralized the attention towards non-Newtonian fluid flow over a Riga-plate. This analysis emphasizes the mechanical properties of Casson fluid flow past a Riga- plate subject to chemical reaction and thermal radiation. The remarkable results have been encapsulated as follows.

- Velocity of the flow field is elevated, as the Lorentz force pointing towards the mainstream velocity. The reverse trend is noticed for opposing flow.

- The boundary layer separation has been controlled for elevated values of $P$ and distance from the leading edge.

- Improved radiation parameter escalates the rate of heat transfer.

- Raising the values of permeability parameter increases the flow speed and wall shear stress.

- Local and mean mass transfer rate is boosted up for higher values of Schmidt number, although the thickness of concentration boundary layer is reduced.

- Larger Casson parameter and magnetic parameter enhance the mass diffusion where as the unimolecular chemical reaction weakens it.

\section{ACKNOWLEDGEMENTS}

One of the authors, K. Deepa, immensely expresses the gratitude to Anna University, Chennai, for supporting this research work through "Anna Centenary Research Fellowship."

\section{REFERENCES}

Abbas, T., Hayat T., Ayub M., Bhatti M.M. and Asaedi A. (2017) "Electromaganetohydrodynamic nanofluid flow past a porous Riga plate containing gyrotactic microorganism," Neural Comput. Appl., 31, 1905-1913.

Ahmad, A. (2019) "Flow control of non-Newtonian fluid using Riga-plate: Reiner-Phillipoff and PowellEyring viscosity models," J. Appl. Fluid Mech., 12, 127-133.

Al-Habahbeh, O.M., Al-Saqqa M., Safi M. and Abo Khater T. (2016) "Review of magnetohydrodynamic pump applications," Alexandria Engineering Journal, 55, 1347-1358.

Ayub, M., Abbas T. and Bhatti M.M. (2016) "Inspiration of slip effects on electromagnetohydrodynamics (EMHD) nanofluid flow through a horizontal Rigaplate," Eur. Phys. J., 131, 193.

Brewster, M.Q. (1992) Thermal radiative transfer and properties, Wiley.

Carnahan, B., Luther H.A. and Wilkes J.O. (1969) Applied Numerical methods, John Wiley and Sons, New York.

Gebhart, B. and Pera L. (1971) "The nature of vertical natural convection flows resulting from the combined buoyancy effects of thermal and mass diffusion," Int. J. Heat Mass Tran., 14, 2025-2050.

Hayat, T., Ullah I. and Alsaedi A. (2018) "Simultaneous effects of nonlinear mixed convection and radiative flow due to Riga-plate with double stratification," J. Heat Transf., 140.

Iqbal, Z., Azhar E., Mehmood Z. and Maraj E.N. (2017) "Unique outcomes of internal heat generation and thermal deposition on viscous dissipative transport of viscoplastic fluid over a Riga-Plate," Commun. in Theor. Phys., 69, 68-76.

Magyari, E. and Pantokratoras A. (2011) "Aiding and opposing mixed convection flows over the Rigaplate," Commun. Nonlinear Sci. numer. Simult., 16, 3158-3167.

Mustafa, M., Hayat T., Pop I. and Aziz A. (2011) "Unsteady boundary layer flow of a Casson fluid due to an impulsively started moving flat plate," Heat Transfer-Asian Research, 40, 563-576.

Oka, S. (1981)Cardiovascular Hemorheology, Cambridge University Press, New York.

Pantokratoras, A. (2008) "Some exact solutions of boundary layer flows along a vertical plate with buoyancy forces combined with Lorentz forces under uniform suction," Math. Probl. Eng., 2008, 118.

Pantokratoras, A. and Magyari E. (2009) "EMHD freeconvection boundary-layer flow from a Riga-plate," J. Eng. Math., 64,303-315.

Rao, M.E. (2018) "The effects of thermal radiation and chemical reaction on MHD flow of a Casson fluid over and exponentially inclined stretching surface," Journal of Physics Conf. Series, 1000, 012158

Rasool, G. and Zhang T. (2019) "Characteristics of chemical reaction and convective boundary conditions in Powell-Eyring nanofluid flow along a radiative Riga- plate," Heliyon, 5, e01479.

Reza, M., Chahal R. and Sharma N. (2016) "Radiation Effect on MHD Casson Fluid Flow over a PowerLaw Stretching Sheet with Chemical Reaction," International Journal of Chemical and Molecular Engineering, 10 585-590.

Schlichting, H. (1979)Boundary Layer Theory, Wiley, New York.

Shateyi, S., Mabood F. and Lorenzini G. (2017) “Casson fluid flow free convective heat and mass transfer over an unsteady permeable stretching surface considering viscous dissipation," J. Eng. Thermophys., 26, 39-52.

Srinivas, S., Kumar C.K. and Reddy A.S. (2018) "Pulsating flow of Casson fluid in a porous channel with thermal radiation, chemical reaction and applied magnetic field," Nonlinear Analysis: Modelling and Control, 23, 213-233.

Weier, T., Gerbeth G., Mutschke G., Lielausis O. and Platacis E. (2001) "Boundary layer control by means of wall parallel Lorentz forces," Magnetohydrodynamics, 37, 177-186.

\section{LIST OF SYMBOLS}

$a^{\prime} \quad$ Absorption coefficient

$C$ Dimensionless concentration

$C_{p} \quad$ Specific heat

$F \quad$ Wall parallel Lorentz force 
Gr Thermal Grashof number

Gc Solutal Grashof number

$g$ Gravitational acceleration

Jo Current density

$K^{*} \quad$ Chemical reaction parameter

$I \quad$ Width of the magnets and electrodes

$M \quad$ Magnetic parameter

$M_{0} \quad$ Magnetization of the magnets

$N \quad$ Radiation parameter

$P \quad$ Modified Hartmann number

Pr Prandtl number

$P_{y} \quad$ Yield stress of the fluid

Sc Schmidt number

$t \quad$ Dimensionless time

$T$ Dimensionless temperature

$U$ Dimensionless vertical velocity component

$V \quad$ Dimensionless horizontal velocity component

$X \quad$ Dimensionless vertical space coordinate

$Y \quad$ Dimensionless transverse coordinate
Greek symbols

$\alpha \quad$ Thermal diffusivity

$\beta \quad$ Casson parameter

$\beta^{\prime} \quad$ Thermal expansion coefficient

$\beta^{*} \quad$ Volumetric coefficient of expansion with concentration

$\kappa \quad$ Thermal conductivity

$\mu_{b} \quad$ Plastic dynamic viscosity

$\rho \quad$ Density

$v \quad$ Kinematic viscosity

$\sigma \quad$ Electrical conductivity

$\sigma^{*} \quad$ Stefan-Boltzmann constant

$\lambda$ Permeability parameter

$\lambda^{*} \quad$ Permeability

Received: April 24, 2018

Sent to Subject Editor: May 5, 2019

Accepted: August 30, 2019

Recommended by Subject Editor: Fabio Giannetti 